\title{
BMJ
}

\section{Effectiveness of ASO3 adjuvanted pandemic H1N1 vaccine: case-control evaluation based on sentinel surveillance system in Canada, autumn 2009}

\begin{abstract}
Danuta M Skowronski, epidemiologist,,2 Naveed Z Janjua, epidemiologist,,2 Gaston De Serres, epidemiologist, ${ }^{3}$ Travis S Hottes, epidemiologist, ${ }^{1}$ James A Dickinson, professor, ${ }^{4}$ Natasha Crowcroft, director, surveillance and epidemiology, ${ }^{5,6}$ Trijntje L Kwindt, research assistant/data coordinator, ${ }^{1}$ Patrick Tang, medical microbiologist, ${ }^{1,7}$ Hugues Charest, virologist, ${ }^{3}$ Kevin Fonseca, clinical virologist, ${ }^{8}$ Jonathan B Gubbay, medical microbiologist, ${ }^{6,9}$ Nathalie Bastien, virologist, ${ }^{10}$ Yan Li, virologist, ${ }^{10}$ Martin Petric, clinical virologist ${ }^{1,7}$
\end{abstract}

${ }^{1}$ British Columbia Centre for Disease Control, Vancouver, BC, Canada

${ }^{2}$ University of British Columbia, School of Population and Public Health, Vancouver

${ }^{3}$ Institut National de Santé Publique du Québec, 2400 d'Estimauville, Québec, QC,

Canada

${ }^{4}$ Departments of Family Medicine and Community Health Sciences, University of Calgary, Calgary, AB, Canada

${ }^{5}$ Ontario Agency for Health Protection and Promotion, Toronto, ON, Canada

${ }^{6}$ Department of Laboratory Medicine and Pathobiology, Faculty of Medicine, University of Toronto, Toronto

${ }^{7}$ Department of Pathology and Laboratory Medicine, University of British Columbia, Vancouver

${ }^{8}$ Alberta Provincial Laboratory, Calgary

${ }^{9}$ Ontario Agency for Health Protection and Promotion, Public Health Laboratory, Toronto

${ }^{10}$ Public Health Agency of Canada, National Microbiology Laboratory, Winnipeg, MB, Canada

Correspondence to: $\mathrm{D}$

M Skowronski, Epidemiology Services, BC Centre for Disease Control, 655 West 12th Avenue, Vancouver, BC, Canada V5Z 4R4 danuta.skowronski@bccdc.ca

Cite this as: $B M J$ 2011;342:C7297 doi:10.1136/bmj.c7297

\section{ABSTRACT}

Objective To assess the effectiveness of the pandemic influenza A/H1N1 vaccine used in Canada during autumn 2009.

Design Test negative incident case-control study based on sentinel physician surveillance system.

Setting Community based clinics contributing to sentinel networks in British Columbia, Alberta, Ontario, and Quebec, Canada.

Participants 552 patients who presented to a sentinel site within seven days of onset of influenza-like illness during the primary analysis period between 8 November and 5 December 2009; participants were mostly ( $280 \%)$ children and adults under 50 years old.

Interventions Monovalent AS03 adjuvanted pandemic influenza $A / H 1 N 1$ vaccine as the predominant formulation (>95\%) distributed in Canada.

Main outcome measures Vaccine effectiveness calculated as 1-(odds ratio for influenza in vaccinated (received pandemic H1N1 vaccine at least two weeks before onset of influenza-like illness) versus unvaccinated participants), with adjustment for age, comorbidity, province, timeliness of specimen collection, and week of illness onset. Sensitivity analyses explored the influence of varying analysis periods between 1 November and 31 December, receipt of trivalent seasonal influenza vaccine, and restriction to participants without comorbidity.

Results During the primary analysis period, pandemic H1N1 was detected by reverse transcription polymerase chain reaction in 209/552 (38\%) participants; rates were highest in children and young adults (40\%) and lowest in people aged 65 or over (9\%). Among the 209 cases, 35 (17\%) reported comorbidity compared with $80 / 343$ (23\%) controls. Two (1\%) cases had received pandemic H1N1 vaccine at least two weeks before the onset of illness, compared with 58/343 (17\%) controls, all single dose. Adjusted vaccine effectiveness overall was $93 \%$ ( $95 \%$ confidence interval $69 \%$ to $98 \%$ ). High estimates of vaccine protection-generally at least $90 \%$-were maintained across most sensitivity analyses.

Conclusions Although limited by a small number of vaccine failures, this study suggests that the monovalent AS03 adjuvanted vaccine used in Canada during autumn 2009 was highly effective in preventing medically attended, laboratory confirmed pandemic H1N1 illness, with reference in particular to a single dose in children and young adults.

\section{INTRODUCTION}

In Canada, public health authorities sought to immunise the population against pandemic influenza A/H1N1 during the autumn of 2009. They selected a monovalent AS03 adjuvanted product as the primary formulation for distribution in Canada (about 23 million doses; 95\%). A second unadjuvanted product made up most of the remaining vaccine distributed (about 1.1 million doses; $<5 \%$ ) and was targeted primarily at women in the first half of pregnancy. Both products were domestically manufactured in Laval, Quebec, Canada and distributed by GlaxoSmithKline. A small quantity of unadjuvanted vaccine was also supplied by CSL Biotherapies from Australia (about 200000 doses; $<1 \%$ ). Canada procured enough pandemic vaccine to immunise the entire population free of charge. Supply was initially limited, however, requiring sequencing of roll-out of vaccine (web appendix A). In participating provinces, the first target groups - typically consisting of very young children, pregnant women, and people aged over 65 with comorbidity-began receiving vaccine on 26 October 2009 (week 43). Vaccine was made available to sequential groups over the ensuing four weeks and finally to all members of the population starting from 19-25 November 2009 (weeks 46-47). Some people who did not belong to sequenced groups may have received vaccine before this date, at the discretion of immunisation providers. 
Pre-marketing immunogenicity trials in Europe, Japan, and North America showed that a single dose of the AS03 adjuvanted vaccine produced in Canada was highly immunogenic. ${ }^{1}$ Fold rises in geometric mean titres after a single dose were substantially above thresholds specified by the European Agency for the Evaluation of Medicinal Products (EMEA) for approval of seasonal vaccines. ${ }^{2}$ Results from these trials among population groups including children, young adults, and adults aged over 60 showed seroprotection rates exceeding $90 \% .{ }^{134} \mathrm{~A}$ randomised trial of children aged 6 months to 12 years $(n=937)$ showed that $\mathrm{AS} 03$ adjuvanted pandemic $\mathrm{H} 1 \mathrm{~N} 1$ vaccine was more immunogenic than a non-adjuvanted whole virus formulation, especially among children under 3 years of age. ${ }^{5}$

One the basis of these findings, health authorities anticipated that a single dose of the AS03 adjuvanted pandemic $\mathrm{H} 1 \mathrm{~N} 1$ vaccine administered in Canada would be highly protective in all age groups. Several caveats to the interpretation of immunogenicity data exist, however. Firstly, quantification of immunogenicity is typically based on the haemagglutination inhibition assay, which may not be an accurate measure of functional antibody response. ${ }^{6}$ Secondly, seroprotective thresholds cited by the EMEA for the approval of seasonal influenza vaccines have not been specifically assessed for the novel pandemic virus. ${ }^{2}$ Thirdly, trials of immunogenicity assess antibody response to the specific reference strain of vaccine selected as that considered most likely to dominate. The pool of circulating viruses may instead include a mix of variants, resulting in reduced clinical effectiveness. Although viruses detected globally through the 2009-10 influenza surveillance period mostly retained a close match to the pandemic vaccine component, ${ }^{7}$ sampling may not be fully representative of circulating strains. Finally, the number of participants included in premarketing immunogenicity trials, especially among children, was small and details of the characteristics of participants were inadequate to enable broad and reliable generalisation to the full population immunised. ${ }^{134}$

For the above reasons, approval of pandemic vaccine based on pre-marketing immunogenicity results was accompanied in several countries by the expectation that the effectiveness of the vaccine would also be assessed with post-marketing epidemiological methods. Here, we report estimates of the effectiveness of the AS03 adjuvanted pandemic $\mathrm{H} 1 \mathrm{~N} 1$ vaccine most used in Canada during the autumn of 2009 based on Canada's well established sentinel surveillance system and predicated on a test negative incident case-control design.

\section{METHODS}

Sentinel vaccine effectiveness monitoring system

As previously described, networks of sentinel physicians in British Columbia, Alberta, Quebec, and Ontario contribute to influenza surveillance and monitoring of vaccine effectiveness annually in Canada. ${ }^{8-11}$
During the pandemic, and as part of surveillance activities, sentinel physicians were specifically exempt from restrictions on testing that applied to clinicians outside the network. Sentinel physicians are provided with kits for monitoring vaccine effectiveness to submit respiratory specimens (nasal or nasopharyngeal) for influenza testing accompanied by epidemiological information collected from eligible consenting patients. Patients are eligible if they present to one of more than 500 contributing community based physicians within seven days of onset of influenza-like illness. Influenza-like illness is defined as the acute onset of fever and cough and one or more of sore throat, myalgia, arthralgia, and prostration. Clinical discretion is incorporated into the decision to test. All participants gave informed oral consent to be included in vaccine effectiveness monitoring, and this was documented on the requisition. Only specimens accompanied by confirmation of consent and complete information on the questionnaire are included in the analysis of vaccine effectiveness.

Information collected about immunisation status included the type (adjuvanted or unadjuvanted) and number of doses of 2009-10 pandemic vaccine received and whether the last dose was at least two weeks before the onset of illness. The questionnaire also captured the presence (yes/no to any) of one or more of the following high risk chronic conditions defined by the National Advisory Committee on Immunization: heart, lung, renal, metabolic, blood, and immune conditions or conditions that compromise the management of respiratory secretions and increase the risk of aspiration. ${ }^{12}$ Information on receipt of trivalent inactivated influenza vaccine during the 2009-10, 2008-9, or 2007-8 seasons was also collected. Immunisation registries do not exist in most provinces of Canada, so the vaccine status based on patients' report cannot be further verified.

\section{Vaccines}

Arepanrix (GlaxoSmithKline) is a monovalent AS03 adjuvanted and formalin inactivated deoxycholate split virion product manufactured in Laval, Quebec. It made up about $95 \%$ of the pandemic $\mathrm{H} 1 \mathrm{~N} 1$ vaccine distributed in Canada during the autumn 2009 immunisation campaign. A $0.5 \mathrm{~mL}$ dose of Arepanrix contained $3.75 \mu \mathrm{g}$ haemagglutinin derived from A/California/7/2009,NYMC X-179A as the representative pandemic influenza $\mathrm{A} / \mathrm{H} 1 \mathrm{~N} 1$ antigen and was administered intramuscularly. ${ }^{1}$ For this vaccine, adjuvant and antigen were packaged separately and required mixing before administration. The product monograph specified a $0.25 \mathrm{~mL}$ dose of the adjuvanted vaccine for children aged 6 months to 9 years and a 0.5 $\mathrm{mL}$ dose for older children and adults. ${ }^{1}$ National guidance documents initially suggested a two dose schedule spaced three weeks apart for children aged 6 months to 9 years, ${ }^{13}$ but decisions about that were subsequently adjusted at the provincial level owing to the availability, sequencing, and roll-out of vaccine in relation to the evolving epidemic curve. All 


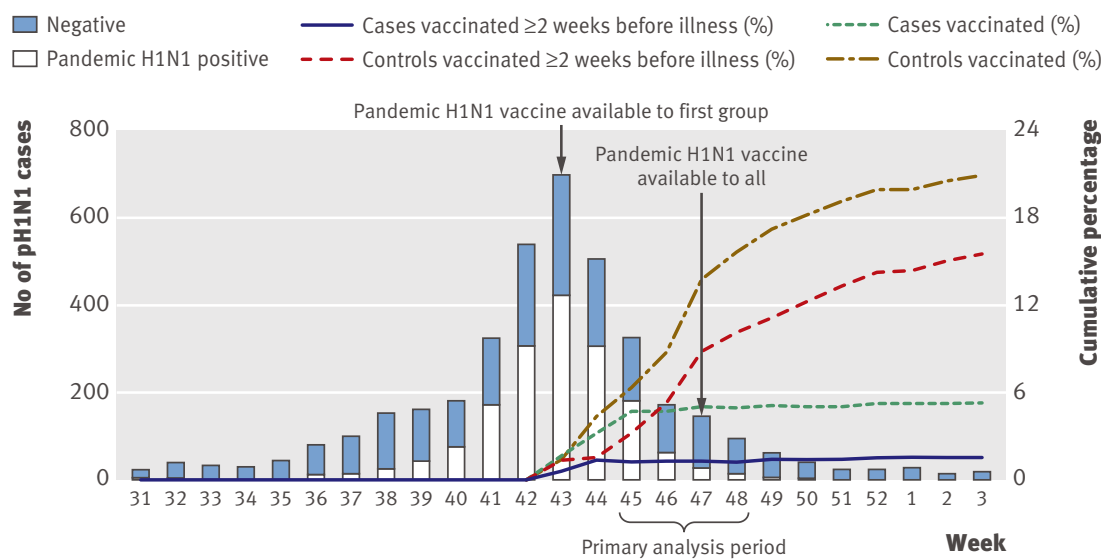

Pandemic H1N1 detections, and cumulative proportion vaccinated against pandemic H1N1 among cases and controls, by week $(n=3898)$. Excludes specimens from patients who failed to meet the influenza-like illness case definition or had unknown status; specimens collected > 7 days after influenza-like illness onset or with unknown interval; and patients with chronic conditions unknown, age unknown, or influenza test results unavailable or inconclusive on typing. One specimen positive for seasonal A/H1N1 (week 46) and four positive for seasonal A/H3N2 (weeks 33, 41, 44, and 50) are not represented on graph. Primary analysis period captures week 45 (8 November) to week 48 (5 December). Secondary analysis period captures week 44 (1 November) to week 52 (31 December). Cumulative percentages for patients vaccinated $\geq 2$ weeks before illness onset exclude from numerator and denominator those vaccinated $<2$ weeks before illness onset. Figure shows cumulative percentages vaccinated from week 43 that are not directly comparable to proportions shown in table 3 for weeks $45-48$

unadjuvanted vaccines used in Canada were also monovalent and formalin inactivated, sodium deoxycholate or sodium taurodeoxycholate (CSL Panvax) split products, ${ }^{14-16}$ containing $15 \mu \mathrm{g}$ haemagglutinin of the same antigen per $0.5 \mathrm{~mL}$ dose administered intramuscularly. Seasonal 2009-10 trivalent inactivated influenza vaccine, supplied by two manufacturers (domestically produced Fluviral, GlaxoSmithKline; and imported Vaxigrip, Sanofi-Pasteur), was also available in Canada. ${ }^{178}$ As recommended by the World Health Organization, the 2009-10 seasonal vaccine contained $15 \mu \mathrm{g}$ haemagglutinin as split product derived from each of A/Brisbane/59/2007(H1N1)-like, A/Brisbane/10/2007(H3N2)-like, and B/Brisbane/60/2008 (Victoria lineage)-like antigens. ${ }^{19}$ Administration of seasonal influenza vaccine was delayed in most provinces of Canada pending the availability of pandemic vaccine, but it could be administered concomitantly in the opposite limb. ${ }^{12}$

\section{Laboratory identification}

The provincial laboratory of each participating province tested for influenza viruses by real time reverse transcription polymerase chain reaction according to established protocols (web appendix B).

\section{Vaccine effectiveness analysis}

We estimated the effectiveness of vaccine by the test negative incident case-control design as previously described. ${ }^{1011}$ Cases were participants in whom pandemic influenza A/H1N1 was detected; controls tested negative for influenza $\mathrm{A}$ and $\mathrm{B}$. Because influenza vaccine is recommended only for people aged at least 6 months, younger participants and those with information on age missing were excluded. A consensus view is that a period of at least 14 days is needed to achieve a protective concentration of antibody after influenza vaccination, but this timeline may vary. ${ }^{20}$ Because the question related to the interval between onset of influenza-like illness and immunisation was dichotomised at two weeks, we could not further explore the timeline to protection. We thus excluded patients immunised less than two weeks before the onset of illness. However, we also explored vaccine effectiveness by including these patients in the vaccinated and unvaccinated categories.

Vaccine became available during week 43, and influenza activity peaked in week 44 with little activity after week 48 (figure). We thus selected weeks $45-48$ as the primary analysis period. Because uptake of pandemic vaccine was evolving over the analysis period (figure), estimates of coverage cannot be derived from this study. For this reason also, we explored an element of time in models of estimates of vaccine effectiveness.

We used logistic regression to estimate odds ratios for the effect of pandemic H1N1 vaccine on medically attended, laboratory confirmed pandemic influenza A/H1N1 infection overall and among participants under 50 years of age through sequential models with adjustment for age (6 months to 9 years, 10-19 years, 20-49 years, and $\geq 50$ years), chronic conditions (yes/ no), province (British Columbia, Alberta, Ontario, or Quebec), timeliness of medical visit $(\leq 4$ days or $>4$ days from the onset of influenza-like illness), and week of onset of illness. We chose age categories on the basis of recommendations on paediatric vaccine dose/schedule that differed above and below 9 years of age, as well as considerations related to pandemic H1N1 risk differential, recognising that fewer cases were reported among older adults. Sex was not influential and was not included in any of the models. We calculated vaccine effectiveness as (1-odds ratio) $\times 100 .{ }^{21}$ In sensitivity analyses, we explored the effect of varying analysis periods between weeks 44 and 52, varying definitions of vaccine status based on interval in relation to illness onset, adjustment for day (rather than week) of illness onset, receipt of seasonal influenza vaccine, and restriction to participants without comorbidity.

\section{RESULTS}

\section{Influenza profile}

The figure shows the epidemic curve of influenza test result by onset of illness. During the primary analysis period between week 45 (8 November) and week 48 (5 December), 867 specimens were submitted. Because of a delay in submission for ethics board approval in Ontario, sentinel physicians in that province did not have access to the revised 2009-10 questionnaire until after 20 November (week 47). For this reason, a substantial proportion of specimens from Ontario were submitted without pandemic $\mathrm{H} 1 \mathrm{~N} 1$ vaccine status recorded and were thus excluded from the analysis. Overall characteristics of patients excluded owing to missing pandemic $\mathrm{H} 1 \mathrm{N1}$ vaccination status were 
Table 1 Inclusion and exclusion of participants for specimens submitted during primary analysis period spanning onset of illness from week 45 (8 November 2009) to week 48 (5 December 2009)

\begin{tabular}{|c|c|c|}
\hline Exclusion criteria & No excluded & No included \\
\hline Specimen submitted from consenting patients & 0 & 866 \\
\hline Influenza-like illness presentation not met or unknown & 31 & 835 \\
\hline $\begin{array}{l}\text { Specimen collected }>7 \text { days after onset of influenza-like illness* } \\
\text { or interval unknown }\end{array}$ & 22 & 813 \\
\hline Chronic conditions unknown & 63 & 750 \\
\hline Age unknown or $<6$ months & 7 & 743 \\
\hline Influenza RT-PCR results unavailable or inconclusive & 6 & 737 \\
\hline Pandemic H1N1 vaccine status missing or unknown & $136 \dagger$ & 601 \\
\hline $\begin{array}{l}\text { Pandemic H1N1 vaccine received }<2 \text { weeks before onset } \\
\text { of influenza-like illness }{ }^{\star}\end{array}$ & 49 & 552 \\
\hline Contributed to analysis & 0 & 552 \\
\hline
\end{tabular}

RT-PCR=reverse transcription polymerase chain reaction.

*Defined as acute onset of fever and cough and one or more of sore throat, arthralgia, myalgia, and prostration. +Because of delay in submission for annual ethics approval in Ontario, sentinel physicians in that province did not have access to revised 2009-10 questionnaire until after 20 November (week 47); 75/122 of specimens from Ontario had unknown pandemic H1N1 vaccine status and were excluded from analysis.

similar to those of patients included in the analyses (data not shown). After we applied inclusion and exclusion criteria, 552 participants were available for the primary analysis period (table 1 ).

Overall during the primary analysis period, 209 $(38 \%)$ specimens were positive for pandemic $\mathrm{H} 1 \mathrm{~N} 1$ and no seasonal strains were detected (table 2). Influenza positivity varied by province and by week of analysis; 145/263 (55\%), 57/144 (40\%), 20/92 (22\%), and 14/67 (21\%) sentinel specimens were positive over successive weeks 45-48. During the extended secondary analysis period spanning weeks 44-52, 993 specimens met the inclusion criteria, pandemic $\mathrm{H} 1 \mathrm{~N} 1$ was diagnosed in $411(41 \%)$, and $\mathrm{A} / \mathrm{H} 3 \mathrm{~N} 2$ was detected in 2 $(0.2 \%)$ specimens, both from Quebec. No other seasonal influenza viruses were detected.

\section{Participant profile}

Table 3 shows the distribution of detection of pandemic A/H1N1 by sex, age, province, chronic conditions, and vaccine status. The distribution of characteristics was similar between the primary and secondary analysis periods (not shown). Young adults aged 20-49 years made up the greatest proportion of participants $(44 \%)$, followed by children aged 6 months to 19 years $(39 \%)$; fewer older adults aged $50-64(12 \%)$ or 65 and over $(4 \%)$ were included. The proportion of patients who tested positive for pandemic $\mathrm{H} 1 \mathrm{~N} 1$ varied by age; it was highest in those aged $10-19$ years $(42 \%), 6$ months to 9 years $(41 \%)$, and $20-49$ years $(40 \%)$; lower in adults aged $50-64$ years $(30 \%)$; and lowest in people aged 65 years or over $(9 \%)(\mathrm{P}=0.02)$. Overall, cases were slightly younger than controls. The proportion with chronic conditions was slightly higher among controls than among cases $(23 \%$ v $17 \% ; \mathrm{P}=0.06)$ and increased with age but was generally comparable to the proportion observed for controls during previous seasons (14-23\%) and to estimates from the Canadian Community Health Survey
(15-20\%). ${ }^{9-1122}$ The proportion immunised with 2008-9 seasonal vaccine among controls $(28 \%)$ was slightly lower than but comparable to that reported in a previous publication (33\%) and by the Canadian Community Health Survey (32\%). ${ }^{1123}$ A detailed comparison of characteristics between controls who did and did not receive the pandemic $\mathrm{H} 1 \mathrm{~N} 1$ vaccine is also shown in web appendix $\mathrm{C}$.

Pandemic H1N1 was detected in a greater proportion of specimens collected within four days of onset of illness $(190 / 487 ; 39 \%)$ compared with specimens collected at $5-7$ days $(19 / 65 ; 29 \%)(\mathrm{P}=0.13)$. The proportion of cases $(190 / 209 ; 91 \%)$ and controls $(297 / 343$; $87 \%$ ) whose specimens were collected at or before four days was comparable $(\mathrm{P}=0.13)$ and did not vary among those with $(99 / 115 ; 86 \%)$ or without (388/ 437; 89\%) chronic conditions $(\mathrm{P}=0.42)$; it did vary slightly among vaccinated $(48 / 60 ; 80 \%)$ compared with unvaccinated participants $(439 / 492 ; 89 \%)$ $(\mathrm{P}=0.036)$ (table 3).

\section{Immunisation profile}

During the primary analysis period, 11\% (60/552) of participants reported receipt of pandemic $\mathrm{H} 1 \mathrm{~N} 1 \mathrm{vac}-$ cine at least two weeks before onset of illness. The proportion who reported receipt of pandemic H1N1 vaccine varied with age and comorbidity (table 3 ). Information on the type of vaccine received was recorded by only $21 / 60(35 \%)$ vaccine recipients, but all reported receiving the adjuvanted formulation. During the secondary analysis period, 52/108 (48\%) vaccinated participants reported the type of vaccine they had received, and of these $96 \%(50 / 52)$ reported receiving the adjuvanted formulation. During the primary analysis period, all vaccinated participants reported having received a single dose. During the secondary analysis period, two children (4 and 8 years of age) reported two dose immunisation.

During the primary analysis period, just two vaccinated cases were identified (table 3). One of the two vaccine failures was a healthy 9 year old child from Quebec with onset of influenza-like illness in midNovember. The other was a young adult with underlying comorbidity from British Columbia with onset of illness in the second half of November. Only 29/517 $(6 \%)$ participants reported receipt of the 2009-10 seasonal vaccine, and of these $13(45 \%)$ had also received the pandemic $\mathrm{H} 1 \mathrm{~N} 1$ vaccine.

\section{Vaccine effectiveness estimates}

Table 4 provides estimates of vaccine effectiveness overall and among participants under 50 years of age for the primary analysis period with and without adjustment, followed by sensitivity analyses and restrictions applied to that period. It also gives estimates for varying intervals within the secondary analysis period. The crude estimate of vaccine effectiveness during the primary analysis period was $95 \%$ (95\% confidence interval $80 \%$ to $99 \%$ ), and this changed only slightly with full adjustment to $93 \%$ (69\% to $98 \%$ ). 
Table 2 | Laboratory profile of specimens included during primary analysis period spanning onset of illness from week 45 (8 November 2009) to week 48 (5 December 2009). Values are numbers (percentages)

\begin{tabular}{|c|c|c|c|c|c|}
\hline Specimen included & Alberta $(n=96)$ & British Columbia $(n=209)$ & Ontario $(n=33)$ & Quebec $(n=217)$ & Total $(n=555)$ \\
\hline Influenza negative & $66(69)$ & $133(64)$ & $31(94)$ & $113(52)$ & $343(62)$ \\
\hline Influenza positive: & $30(31)$ & $76(36)$ & $2(6)$ & $104(48)$ & $212(38)$ \\
\hline A positive & $30(100)$ & $76(100)$ & $2(100)$ & $104(100)$ & $212(100)$ \\
\hline B positive & $0(0)$ & $0(0)$ & $0(0)$ & $0(0)$ & $0(0)$ \\
\hline \multicolumn{6}{|l|}{ Influenza A positive: } \\
\hline $\mathrm{A} / \mathrm{H} 1 \mathrm{~N} 1$ & $0(0)$ & $0(0)$ & $0(0)$ & $0(0)$ & $0(0)$ \\
\hline $\mathrm{A} / 3 \mathrm{~N} 2$ & $0(0)$ & $0(0)$ & $0(0)$ & $0(0)$ & $0(0)$ \\
\hline Pandemic A/H1N1 & $30(100)$ & $76(100)$ & $2(100)$ & $101(97)$ & 209 (99) \\
\hline Subtype unknown & $0(0)$ & $0(0)$ & $0(0)$ & $3(3)$ & $3(1)$ \\
\hline
\end{tabular}

High estimates of vaccine protection, generally at least $90 \%$, were robust and maintained across most sensitivity analyses. Altering the paediatric age categories selected for adjustment to include preschool children and younger and older school age children did not meaningfully alter estimates of vaccine effectiveness (available on request). The week (or date) of onset of illness was the only covariate that influenced estimates of vaccine effectiveness. As anticipated, an interval after immunisation was needed for optimal protection. If participants who had received vaccine within two weeks of onset of illness were returned to the analysis as vaccinated, estimates dropped considerably to $55 \%$ $(20 \%$ to $75 \%)$. If those vaccinated within two weeks were instead considered to be unvaccinated, fully adjusted vaccine effectiveness was the same as in the primary analysis. Receipt of 2008-9 or 2009-10 seasonal vaccine had minimal influence on the effectiveness of pandemic $\mathrm{H} 1 \mathrm{~N} 1$ vaccine in multivariable analysis.

Fully adjusted estimates of vaccine effectiveness were lower $(78 \%, 53 \%$ to $90 \%)$ during the secondary analysis period spanning weeks 44-52. We had anticipated this, given that roll-out of vaccine had begun only one week earlier and a larger number of controls without opportunity to receive vaccine were included in week 44 (figure). Estimates of vaccine effectiveness increased during the secondary analysis period when restriction started instead at week $45(87 \%, 62 \%$ to 95\%) and were again comparable to the primary analysis period with later restriction or for weeks 46-47.

\section{DISCUSSION}

Using the sentinel physician surveillance system in Canada, we measured excellent protection conferred by a single dose of the monovalent AS03 adjuvanted pandemic vaccine during the autumn 2009 epidemic in Canada. Specifically, 14 days or more after vaccination, we estimated vaccine effectiveness of 93\% (95\% confidence interval $69 \%$ to $98 \%$ ) against medically attended, laboratory confirmed pandemic influenza A/H1N1 illness. This estimate was robust and maintained $(\geq 90 \%)$ across most sensitivity analyses and primarily reflects protection conferred to children and young adults.
Comparison with other studies

Our estimates of protection by vaccine are consistent with immunogenicity studies indicating very high antibody response to AS03 adjuvanted pandemic $\mathrm{H} 1 \mathrm{~N} 1$ vaccine across age groups, ${ }^{13-5}$ as well as with the protection that would be expected given excellent match of vaccine to circulating virus. The findings are also consistent with recent estimates of vaccine effectiveness derived in a smaller test negative case-control study among children under 10 years of age in New Brunswick, Canada (28 cases (none vaccinated); 63 controls (24 vaccinated)) given a single dose of the same product $(100 \%, 79.5 \%$ to $100 \%) .{ }^{24}$ Our results are also consistent with study findings based on the screening method in Germany among patients aged 14 years and over given the AS03 adjuvanted pandemic $\mathrm{H} 1 \mathrm{~N} 1$ vaccine manufactured by GlaxoSmithKline in Dresden. ${ }^{25}$ In that study, vaccine effectiveness among participants aged 14-59 years was 96.8\% $(95.2 \%$ to $97.9 \%)$ and slightly lower in those aged 60 years or over $(83.3 \%, 71.0 \%$ to $90.5 \%) .{ }^{25}$ A retrospective cohort study from Scotland across all age groups similarly reported protection by vaccine of $95 \%(76 \%$ to $100 \%)$, but the proportionate mix and type(s) of vaccine used were not specified. ${ }^{26}$ Our estimates are higher than those based on the test negative case-control design applied to a linked monitoring network across seven countries of Europe, in which participants received a mix of adjuvanted and nonadjuvanted formulations; additional methodological differences may also explain their lower estimate of vaccine effectiveness of $71.9 \%(45.6 \%$ to $85.5 \%) .{ }^{27} \mathrm{As}$ with all observational designs, the relative strengths and weaknesses, and potential for bias and confounding, have to be taken into account in interpreting each of these datasets. In particular, distribution of pandemic vaccine was progressing as the autumn H1N1 epidemic was evolving in many countries. Consideration of detail related to the change in immunisation coverage among controls over the course of the analysis period is essential for each of these studies.

\section{Strengths and limitations of study}

Strengths of our study include the almost sole use of a single vaccine formulation-thus simplifying 
Table 3 | Participants' profile for primary analysis period spanning onset of illness from week 45 (8 November 2009) to week 48 (5 December 2009). Values are numbers (column percentages)

\begin{tabular}{|c|c|c|c|}
\hline Covariates & $\begin{array}{l}\text { Pandemic H1N1 test } \\
\text { negative control } \\
(n=343)\end{array}$ & $\begin{array}{l}\text { Pandemic H1N1 test } \\
\text { positive case } \\
(n=209)\end{array}$ & $\begin{array}{c}\text { Overall } \\
(n=552)\end{array}$ \\
\hline \multicolumn{4}{|l|}{ Age category: } \\
\hline 6 months to 9 years & $71(21)$ & $50(24)$ & $121(22)$ \\
\hline 10-19 years & $56(16)$ & $40(19)$ & $96(17)$ \\
\hline 20-49 years & $148(43)$ & $97(46)$ & $245(44)$ \\
\hline 50-64 years & $47(14)$ & $20(10)$ & $67(12)$ \\
\hline$\geq 65$ years & $21(6)$ & $2(1)$ & $23(4)$ \\
\hline Median (range) age (years) & $27((1-88)$ & $22((1-68)$ & $24((1-88)$ \\
\hline Female sex & $200(58)$ & $93(45)$ & $293(53)$ \\
\hline Chronic conditions & $80(23)$ & $35(17)$ & $115(21)$ \\
\hline \multicolumn{4}{|l|}{ Province: } \\
\hline Alberta & 66 (19) & $30(14)$ & $96(17)$ \\
\hline British Columbia & $133(39)$ & $76(36)$ & $209(38)$ \\
\hline Ontario & $31(9)$ & $2(1)$ & $33(6)$ \\
\hline Quebec & $113(33)$ & $101(48)$ & $214(39)$ \\
\hline Pandemic H1N1 vaccinated* & $58(17)$ & $2(1)$ & 60 (11) \\
\hline \multicolumn{4}{|l|}{ 2009-10 seasonal vaccine: } \\
\hline Not vaccinated & $297(93)$ & $191(97)$ & $488(94)$ \\
\hline Vaccinated & $24(7)$ & $5(3)$ & $29(6)$ \\
\hline \multicolumn{4}{|l|}{ 2008-9 seasonal vaccine: } \\
\hline Not vaccinated & $213(72)$ & $158(84)$ & $371(77)$ \\
\hline Vaccinated $\dagger$ & $83(28)$ & $29(16)$ & $112(23)$ \\
\hline \multicolumn{4}{|l|}{ Specimen collection interval: } \\
\hline$\leq 4$ days after illness onset & $297(87)$ & 190 (91) & $487(88)$ \\
\hline 5-7 days after illness onset & $46(13)$ & $19(9)$ & $65(12)$ \\
\hline Median (range) days & $2(0-7)$ & $2(0-7)$ & $2(0-7)$ \\
\hline \multicolumn{4}{|l|}{ Pandemic H1N1 vaccination by age: } \\
\hline 6 months to 9 years & 22/71 (31) & $1 / 50(2)$ & 23/121 (19) \\
\hline $10-19$ years & 3/56 (5) & $0 / 40(0)$ & 3/96 (3) \\
\hline $20-49$ years & 21/148 (14) & 1/97 (1) & $22 / 245(9)$ \\
\hline $50-64$ years & 11/47 (23) & $0 / 20(0)$ & $11 / 67(16)$ \\
\hline$\geq 65$ years & $1 / 21(5)$ & $0 / 2(0)$ & $1 / 23(4)$ \\
\hline \multicolumn{4}{|l|}{ Chronic conditions by age: } \\
\hline 6 months to 9 years & 11/71 (15) & $6 / 50(12)$ & $17 / 121(14)$ \\
\hline 10-19 years & $8 / 56(14)$ & 9/40 (23) & $17 / 96$ (18) \\
\hline $20-49$ years & 29/148 (20) & 12/97 (12) & $41 / 245(17)$ \\
\hline $50-64$ years & 18/47 (38) & $7 / 20(35)$ & $25 / 67(37)$ \\
\hline$\geq 65$ years & 14/21 (67) & $1 / 2(50)$ & $15 / 23(65)$ \\
\hline \multicolumn{4}{|l|}{ With chronic conditions: } \\
\hline Not pandemic H1N1 vaccinated & $59(74)$ & $34(97)$ & $93(81)$ \\
\hline Pandemic H1N1 vaccinated & $21(26)$ & $1(3)$ & $22(19)$ \\
\hline \multicolumn{4}{|l|}{ Without chronic conditions: } \\
\hline Not pandemic H1N1 vaccinated & $226(86)$ & $173(99)$ & 399 (91) \\
\hline Pandemic H1N1 vaccinated & 37 (14) & $1(1)$ & $38(9)$ \\
\hline \multicolumn{4}{|c|}{ Interval to specimen collection $\leq 4$ days: } \\
\hline Not pandemic H1N1 vaccinated & $251(85)$ & $188(99)$ & $439(90)$ \\
\hline Pandemic H1N1 vaccinated & $46(15)$ & $2(1)$ & $48(10)$ \\
\hline \multicolumn{4}{|c|}{ Interval to specimen collection 5-7 days: } \\
\hline Not pandemic H1N1 vaccinated & $34(74)$ & $19(100)$ & $53(82)$ \\
\hline Pandemic H1N1 vaccinated & $12(26)$ & $0(0)$ & $12(18)$ \\
\hline
\end{tabular}

*Received vaccine $\geq 2$ weeks before onset of illness; excludes from numerator and denominator those immunised $<2$ weeks before onset.

†Excludes children too young to have received 2008-9 seasonal vaccine. interpretation - and a well established system for monitoring of vaccine effectiveness that has been rehearsed across several seasons in Canada (since 2004). ${ }^{8-11}$ In general, adjusted point estimates of the effectiveness of (non-adjuvanted) influenza vaccine against seasonal strains based on this sentinel surveillance system in Canada have ranged between $50 \%$ and $60 \%$ overall. ${ }^{8-11}$ Estimates from the sentinel system have been comparable to available results from randomised controlled trials in the United States using the Canadian GlaxoSmithKline vaccine during the corresponding season (2005-6 and 2006-7) ${ }^{28}$ Estimates of vaccine effectiveness have also been well correlated in component specific analyses with the relative match/mismatch of vaccine antigens to circulating strains. With a good match, such as between the A/H1N1 component of the 2006-7 (non-adjuvanted) vaccine and the then circulating virus (A/NewCaledonia/20/99 (H1N1)-like), vaccine effectiveness was also found to exceed $90 \%$ when measured by this system. ${ }^{10} \mathrm{~A}$ further advantage of the sentinel system is its breadth of representation, including more than 500 sentinel sites spanning four provinces. Such geographical breadth becomes a particular strength in capturing variation in circulating viruses and when pursuing the rare event of vaccine failure in the context of highly immunogenic vaccine. In our analysis, adjustment by week of onset of illness and exploration over varying time periods in sensitivity analyses made the effect of co-evolving epidemic activity and vaccine roll-out explicit.

Limitations of the sentinel system for monitoring vaccine effectiveness have been described previously. ${ }^{8-11} \mathrm{In}$ particular, despite study protocols, standardised questionnaires, instruction kits, and ethics review, the sentinel system remains a public health surveillance approach so that monitoring of vaccine effectiveness is not as rigorous as in controlled clinical trials. The test negative design superimposed on this system is an observational case-control method, and the same epidemiological considerations as with any case-control study still apply. This approach cannot guard against the systematic influences of bias (selection, information) or unmeasured confounders. We carefully assessed participants' profiles against historical and expected demographic information as a flag for possible evidence of selection bias. Despite reassuring profiles, however, this still cannot be fully ruled out. We required participants to have presented to a physician with influenzalike illness, thus standardising in part, but not fully, for healthcare seeking behaviour, access to healthcare, and severity of illness. However, clinicians' discretion in the decision to test is recognised.

Although this was not a blinded clinical trial, we collected information on vaccine before the physician or patient knew the influenza test result, thus helping to reduce recall bias. We have interpreted results as reflecting the AS03 adjuvanted vaccine most used in Canada on the basis of relative distribution and limited access to the unadjuvanted formulation, but most vaccinated participants did not know or report the type of vaccine they received. We collected information on 
Table 4 | Effectiveness of vaccine against medically attended, laboratory confirmed pandemic H1N1, including additional sensitivity analyses

Vaccine effectiveness ${ }^{\star}(95 \% \mathrm{Cl})$ (No of cases; No of controls) Overall Aged $<50$ years

Covariates in primary analysis-weeks 45-48 (8 November to 5 December 2009)

\begin{tabular}{|c|c|c|}
\hline Unadjusted & $95.3(80.3$ to 98.9$)(209 ; 343)$ & $94.6(77.5$ to 98.7$)(187 ; 275)$ \\
\hline Chronic conditions (yes/no) & $95.1(79.5$ to 98.8$)(209 ; 343)$ & $94.6(77.3$ to 98.7$)(187 ; 275)$ \\
\hline Age† & $95.6(81.4$ to 98.9$)(209 ; 343)$ & $95.0(79.0$ to 98.8$)(187 ; 275)$ \\
\hline Province (BC, $A B, O N, Q C)$ & $95.2(80.1$ to 98.9$)(209 ; 343)$ & 94.5 (76.9 to 98.7$)(187 ; 275)$ \\
\hline Specimen collection interval ( $\leq 4 />4$ days) & $95.1(79.9$ to 98.8$)(209 ; 343)$ & $94.6(77.2$ to 98.7$)(187 ; 275)$ \\
\hline Week of illness onset & $93.2(71.5$ to 98.4$)(209 ; 343)$ & $91.5(63.9$ to 98.0$)(187 ; 275)$ \\
\hline Age and chronic conditions $\dagger$ & $95.5(81.1$ to 98.9$)(209 ; 343)$ & $95.0(78.8$ to 98.8$)(187 ; 275)$ \\
\hline Age, chronic conditions, province & $95.5(81.0$ to 98.9$)(209 ; 343)$ & $94.9(78.3$ to 98.8$)(187 ; 275)$ \\
\hline Age, chronic conditions, province, specimen collection interval† & $95.5(80.8$ to 98.9$)(209 ; 343)$ & $94.9(78.2$ to 98.8$)(187 ; 275)$ \\
\hline $\begin{array}{l}\text { Age, chronic conditions, province, specimen collection interval, } \\
\text { week of illness onset† }\end{array}$ & $92.7(68.6$ to 98.3$)(209 ; 343)$ & $91.1(61.5$ to 98.0$)(187 ; 275)$ \\
\hline \multicolumn{3}{|l|}{ Variations applied to primary analysis period (weeks 45-48) } \\
\hline Excluding Ontarioł‡ & $92.2(66.5$ to 98.2$)(207 ; 312)$ & $90.4(57.9$ to 97.8$)(185 ; 253)$ \\
\hline Restricted to only those with no chronic conditions $† \S$ & $95.6(66.8$ to 99.4$)(174 ; 263)$ & $95.3(63.7$ to 99.4$)(160 ; 227)$ \\
\hline Including those with unknown chronic conditions as "no chronic condition" $† \ddagger$ & $92.8(69.4$ to 98.3$)(216 ; 370)$ & $91.1(61.5$ to 97.9$)(192 ; 297)$ \\
\hline Including those with unknown chronic conditions as "with chronic condition" $¥ \ddagger$ & $92.5(67.9$ to 98.2$)(370 ; 216)$ & $91(61$ to 97.9$)(297 ; 192)$ \\
\hline $\begin{array}{l}\text { Age, chronic conditions, province, specimen collection interval, } \\
\text { day of illness onsett }\end{array}$ & $92.3(67.3$ to 98.2$)$ & 90.5 (58.8 to 97.8$)$ \\
\hline As above, restricted to only those with no chronic conditions $† \S$ & $95.3(64.3$ to 99.4$)(174 ; 263)$ & $94.8(60.2$ to 99.3$)(160 ; 227)$ \\
\hline Pandemic H1N1 vaccine status unknown considered as "unvaccinated" $¥ \ddagger$ & $93.1(70.6$ to 98.4$)(214 ; 350)$ & $91.6(63.6$ to 98.0$)(191 ; 281)$ \\
\hline As above, restricted to only those with no chronic conditions $† \S$ & $95.6(66.5$ to 99.4$)(175 ; 270)$ & $95.2(63.4$ to 99.4$)(161 ; 233)$ \\
\hline Pandemic H1N1 vaccine status unknown considered as "vaccinated" $\ddagger \ddagger$ & 75.9 (43.9 to 89.7$)(350 ; 214)$ & $75.6(38.9$ to 90.3$)(281 ; 191)$ \\
\hline As above, restricted to only those with no chronic conditions $\nmid \S$ & $92.0(65.2$ to 98.2$)(270 ; 175)$ & $91.4(62.3$ to 98.0$)(233 ; 161)$ \\
\hline Pandemic H1N1 vaccine interval <2 weeks considered vaccinated†‡ & $55.2(19.8$ to 75.0$)(225 ; 376)$ & $54.6(14.8$ to 75.9$)(201 ; 300)$ \\
\hline As above, restricted to only those with no chronic conditions $† \S$ & $64.0(26.0$ to 82.5$)(184 ; 285)$ & $70.2(34.4$ to 86.4$)(168 ; 246)$ \\
\hline Pandemic H1N1 vaccine interval <2 weeks considered unvaccinated $\ddagger$ & $92.6(68.5$ to 98.3$)(225 ; 376)$ & $91.1(61.3$ to 97.9$)(201 ; 300)$ \\
\hline As above, restricted to only those with no chronic conditions $\nmid \S$ & $95.4(64.7$ to 99.4$)(184 ; 285)$ & $94.9(60.9$ to 99.3$)(168 ; 246)$ \\
\hline $\begin{array}{l}\text { Age, chronic conditions, province, specimen collection interval, week of illness } \\
\text { onset, receipt of } 2008-9 \text { seasonal vaccine† }\end{array}$ & $88.0(46.9$ to 97.3$)(187 ; 296)$ & $85.2(32.9$ to 96.7$)(167 ; 235)$ \\
\hline As above, restricted to only those with no chronic conditions $\nmid \S$ & $92.3(38.4$ to 99.0$)(158 ; 225)$ & $91.8(33.9$ to 99.0$)(144 ; 192)$ \\
\hline $\begin{array}{l}\text { Age, chronic conditions, province, specimen collection interval, week of illness } \\
\text { onset, receipt of 2009-10 seasonal vaccine† }\end{array}$ & $94.8(60.5$ to 99.3$)(196 ; 321)$ & $93.4(48.2$ to 99.2$)(176 ; 256)$ \\
\hline As above, restricted to only those with no chronic conditions $\nmid \S$ & $92.3(39.1$ to 99.0$)(165 ; 246)$ & 91.3 (30.4 to 98.9$)(152 ; 210)$ \\
\hline Restricted to only those with specimen collection interval $\leq 4$ days $+\pi$ & $90.9(60.5$ to 97.9$)(190 ; 297)$ & $88.8(50.6$ to 97.4$)(171 ; 244)$ \\
\hline \multicolumn{3}{|l|}{ Variations in analysis period (illness onset) ${ }^{\star \star}$} \\
\hline Weeks 44-52 (1 November to 31 December 2009)†‡ & $78.4(52.6$ to 90.2$)(411 ; 577)$ & $75.1(44.5$ to 88.8$)(372 ; 470)$ \\
\hline Weeks 45-52 (8 November to 31 December 2009)†‡ & $86.7(61.8$ to 95.4$)(217 ; 457)$ & $84.1(53.5$ to 94.6$)(194 ; 361)$ \\
\hline Weeks 47-52 (22 November to 31 December 2009)†‡ & 92.5 (41.0 to 99.0$)(36 ; 268)$ & $91.7(33.8$ to 99.0$)(29 ; 211)$ \\
\hline Weeks 46-47 (15 November to 28 November 2009)†‡ & $92.6(41.8$ to 99.1$)(65 ; 165)$ & $92.4(39.3$ to 99.0$)(58 ; 138)$ \\
\hline
\end{tabular}

*Vaccine effectiveness $=(1-$ odds ratio $) \times 100$.

†Adjusted for age as 6 months to 9 years, 10-19 years, 20-49 years, and $\geq 50$ years for overall and as 6 months to 9 years, 10-19 years, and 20-49 years for $<50$ years.

$\ddagger$ Adjusted for age, province, chronic conditions (yes/no), interval since influenza-like illness onset ( $\leq 4 />4$ days), and week of illness onset.

$\S$ Adjusted for age, province, interval since influenza-like illness onset, and week of illness onset.

TAdjusted for age, province, chronic conditions, and week of illness onset.

**During secondary analysis period (weeks 44-52), eight cases had received pandemic H1N1 vaccine $>2$ weeks before illness onset, including five children aged 1-9 years and three young adults aged 20-49 years; of these eight pandemic H1N1 vaccine failures, six cases reported no underlying conditions.

covariates known to influence estimates of vaccine effectiveness (age, chronic conditions, timeliness of specimen collection) and adjusted for recognised confounders tested over several years of application of this system in Canada. Some information, such as on chronic conditions, is dichotomised in a yes/no format without further detail provided. Although this may not represent finer differential across specific conditions, little effect of residual confounding has been attributed to aggregation of comorbidity in studies of influenza vaccination. $^{29}$ Ultimately, however, residual confounding cannot be ruled out.

Canada delayed the administration of the 2009-10 seasonal influenza vaccine until pandemic vaccine became available. This prevented us from assessing the effect of its previous administration on response 


\section{WHAT IS ALREADY KNOWN ON THIS TOPIC}

Immunogenicity trials of ASO3 adjuvanted pandemic H1N1 vaccines showed substantial vaccine induced antibody response after a single dose

Interpretation of immunogenicity studies is limited by serological surrogates for protection, small sample sizes, and uncertain relevance if circulating viruses include an unrecognised mix of antigenic variants

\section{WHAT THIS STUDY ADDS}

The effectiveness of the monovalent AS03 adjuvanted pandemic vaccine exceeded $90 \%$ against medically attended, laboratory confirmed pandemic H1N1 illness during autumn 2009 in Canada

The results apply particularly to children and young adults

Although limited by a small number of vaccine failures, results are consistent with the protection expected given very high vaccine induced antibody response and excellent match to circulating virus du Québec. The funders did not have a role in study design, data collection and analysis, decision to publish, or preparation of the manuscript.

Competing interests: All authors have completed the Unified Competing Interest form at www.icmje.org/coi_disclosure.pdf (available on reques from the corresponding author at danuta.skowronski@bccdc.ca) and declare that they had no financial support from commercial entities for the submitted work. DMS was principal investigator on a clinical trial for which influenza vaccine was provided free by Sanofi-Pasteur and has received research grant funding related to influenza from the Canadian Institutes of Health Research, the Public Health Agency of Canada, and the Michael Smith Foundation for Health Research in the past 36 months. GDS has received research grants from GlaxoSmithKline and SanofiPasteur for unrelated studies in the past 36 months. JBG has received a grant from GlaxoSmithKline to assess an unrelated influenza question. The Canadian Institutes of Health research grant provided salary suppor for TLK.

Ethical approval: Although run as a routine surveillance initiative, the sentinel surveillance system follows a study protocol that is approved by the following ethics review committees in participating provinces: University of British Columbia, Vancouver, BC; University of Calgary, Calgary, AB; University of Toronto, Toronto, ON; and Comité d'éthique de santé publique, Québec. All participants gave informed oral consent to be included in vaccine effectiveness monitoring.

Data sharing: No additional data available. surveillance network, the number of vaccine failures identified in this study was small, a limitation difficult to overcome in the context of highly immunogenic vaccine. This precluded stratified analysis (such as age specific estimates of vaccine effectiveness) and may potentially limit the meaningfulness of extensive multivariable adjustment or sensitivity analysis. Given that more than $80 \%$ of our participants were under 50 years of age, our estimates of protection by vaccine cannot be reliably interpreted for older adults. Further evidence for effectiveness in older people is needed.

\section{Conclusions}

We report estimates of vaccine effectiveness suggesting very high protection conferred by a single dose of the monovalent AS03 adjuvanted pandemic vaccine most used in Canada during autumn 2009, with reference in particular to children and young adults. Although limited by a small number of vaccine failures, we interpret these findings to be consistent with indicators of immunogenicity showing very high vaccine induced antibody response and with virological characterisation suggesting an excellent match of vaccine to circulating virus. Our findings are relevant to the evaluation of pandemic vaccination efforts not only in Canada but also in other countries where adjuvanted vaccines were used. Our results may help to inform the further development of influenza vaccine options.

We recognise the invaluable contribution of frontline sentinel physician sites and the coordination and technical support provided by epidemiological and laboratory staff in all participating provinces. Contributors: DMS, NZJ, GDS, TSH, JAD, NC, HC, KF, JBG, and MP designed the study. DMS, NZJ, GDS, TSH, JAD, NC, and TLK organised sites for collection of data. PT, HC, KF, JBG, NB, YL, and MP oversaw laboratory testing. NZJ, DMS, TSH, and GDS guided analyses. NZJ analysed the data. All authors had access to the data (including statistical reports and tables) and can take responsibility for the study. All authors contributed to the writing of the paper and reviewed and approved the final manuscript. DMS is the guarantor

Funding: Funding for this study was provided by the Canadian Institutes of Health Research (ID: TPA-90193), the British Columbia Centre for Disease Control, Alberta Health and Wellness, the Ontario Agency for Health Protection and Promotion, the Ministère de la santé et des services sociaux du Québec, and the Institut national de santé publique
1 GlaxoSmithKline Inc. Product information leaflet: Arepanrix ${ }^{\mathrm{TM}} \mathrm{H} 1 \mathrm{~N} 1$. 2010. www.gsk.ca/english/docs-pdf/Arepanrix 2010.pdf.

2 Committee for Proprietary Medicinal Products. Note for guidance on harmonization of requirements for influenza vaccines. CPMP/BWP/ 214/96 (circular no 96-0666):1-22. 1997. www.emea.europa.eu/ pdfs/human/bwp/021496en.pdf.

3 GlaxoSmithKline Inc. Results summaries: H1N1 Pandemic influenza vaccine. GSK study no 113482 (FLU Q-PAN H1N1-003 PRI). 2010. www.gsk-clinicalstudyregister.com/result_comp_list.jsp? compound $=\mathrm{H} 1 \mathrm{~N} 1+$ Pandemic+Influenza

+VaccinestudyType=All\&phase=All\&population=All\&marketing=All.

4 GlaxoSmithKline Inc. Results summaries: H1N1 Pandemic influenza vaccine. GSK study no 113847 (FLU Q-PAN H1N1-0029). 2010. www. gsk-clinicalstudyregister.com/result_comp_list.jsp? compound $=\mathrm{H} 1 \mathrm{~N} 1+$ Pandemic + Influenza+

Vaccine\&studyType=All\&phase=All\&population=All\&marketing=All.

5 Waddington CS, Walker WT, Oeser C, Reiner A, John T, Wilkins S, et al. Safety and immunogenicity of $\mathrm{ASO}_{\mathrm{B}}$ adjuvanted split virion versus non-adjuvanted whole virion $\mathrm{H} 1 \mathrm{~N} 1$ influenza vaccine in UK children aged 6 months -12 years: open label, randomised, parallel group, multicentre study. BMJ 2010;340:C2649.

6 De Jong JC, Palache AM, Beyer WEP. Haemagglutination-inhibiting antibody to influenza virus. In Brown F, Haaheim LR, Schild GC, eds. Laboratory correlates of immunity to influenza reassessment. Vol 115. Karger, 2003:63-73.

7 World Health Organization. Recommended viruses for influenza vaccines for use in the 2010-2011 northern hemisphere influenza season. 2010. www.who.int/csr/disease/influenza/ 201002 Recommendation.pdf.

8 Effectiveness of vaccine against medical consultation due to laboratory-confirmed influenza: results from a sentinel physician pilot project in British Columbia, 2004-05. Can Commun Dis Rep 2005;31:181-92.

9 Skowronski DM, Masaro C, Kwindt TL, Mak A, Petric M, Li Y, et al. Estimating vaccine effectiveness against laboratory-confirmed influenza using a sentinel physician network: results from the 2005 2006 season of dual A and B vaccine mismatch in Canada. Vaccine 2007;25:2842-51.

10 Skowronski DM, De Serres G, Dickinson J, Petric M, Mak A, Fonseca K, et al. Component-specific effectiveness of trivalent influenza vaccine as monitored through a sentinel surveillance network in Canada, 2006-07. J Infect Dis 2009;199:168-79.

11 Skowronski DM, De Serres G, Crowcroft NS, Janjua NZ, Boulianne N, Hottes TS, et al. Association between the 2008-09 seasonal influenza vaccine and pandemic H1N1 illness during the springsummer 2009: four observational studies from Canada. PLoS Med 2010;7:e1000258.

12 National Advisory Committee on Immunization. Statement on seasonal trivalent inactivated influenza vaccine (TIV) for the 20092010 season. Can Commun Dis Rep 2009;35:1-41.

13 Public Health Agency of Canada. Guidance document on the use of pandemic influenza A (H1N1) 2009 inactivated monovalent vaccine. 2009. www.phac-aspc.gc.ca/alert-alerte/h1n1/vacc/pdf/ monovacc-guide-eng.pdf.

14 GlaxoSmithKline Inc. Product information leaflet: influenza A (H1N1) 2009 pandemic monovalent vaccine (without adjuvant). 2009. www. 
gsk.ca/english/docs-pdf/unadjuvated\%20H1N1\%20(CAPU01-PIL \%2001.1).pdf.

15 Public Health Agency of Canada. Addendum to the October 21, 2009 guidance document on the use of pandemic influenza $A$ (H1N1) 2009 inactivated monovalent vaccine: use of Panvax ${ }^{\circledR}$ H1N1 vaccine (CSL Biotherapies Inc.) (unadjuvanted). 2009. www.phacaspc.gc.ca/alert-alerte/h1n1/vacc/pdf/Panvax-Addendum-eng.pdf.

16 CSL Biotherapies Inc. Panvax ${ }^{\circledR}$ H1N1 vaccine/Panvax ${ }^{\circledR}$ H1N1 vaccine junior: product information. 2009. http://secure.healthlinks.net.au/ content/csl/pi.cfm?product=csppanva11209.

17 GlaxoSmithKline Inc. Product monograph: Fluviral ${ }^{\circledast}$ (2009-2010). 2009. www.gsk.ca/english/docs-pdf/Fluviral_2009_PM.pdf.

18 Sanofi Pasteur SA. Product monograph: Vaxigrip ${ }^{\circledR}$. 2010. www. vaccineshoppecanada.com/secure/pdfs/ca/vaxigrip_e.pdf.

19 World Health Organization. Recommended composition of influenza virus vaccines for use in the 2009-2010 influenza season. 2009. www.who.int/csr/disease/influenza/200902_recommendation. pdf.

20 Cox RJ, Brokstad KA, Zuckerman MA, Wood JM, Haaheim LR, Oxford JS. An early humoral immune response in peripheral blood following parenteral inactivated influenza vaccination. Vaccine 1994;12:993-9.

21 Orenstein WA, Bernier RH, Dondero TJ, Hinman AR, Marks JS, Bart KJ, et al. Field evaluation of vaccine efficacy. Bull World Health Organ 1985;63:1055-68.

22 Statistics Canada. Canadian community health survey (cycle 3.1). Statistics Canada, 2005.

23 Statistics Canada. Influenza immunization coverage by province and age 2008. Table 105-0501. Health indicator profile, annual estimates, by age group and sex, Canada, provinces, territories, health regions (2007 boundaries) and peer groups, occasional
(1209600 series). 2009. http://cansim2.statcan.gc.ca/cgi-win/ cnsmcgi.pgm?Lang=E\&RootDir=CII/\&Array_Pick=1\&Arrayld=105 0501\&C2DB=PRD\&ResultTemplate=CII\%2FCII.

24 Van Buynder PG, Dhaliwal JK, Van Buynder JL, Couturier C, Minville-Leblanc M, Garceau R, et al. Protective effect of single-dose adjuvanted pandemic influenza vaccine in children. Influenza Other Respi Viruses 2010;4:171-8.

25 Wichmann O, Stocker P, Poggensee G, Altmann D, Walter D, Hellenbrand W, et al. Pandemic influenza A (H1N1) 2009 breakthrough infections and estimates of vaccine effectiveness in Germany 2009-2010. Euro Surveill 2010;15:pii=19561.

26 Simpson CR, Ritchie LD, Robertson C, Sheikh A, McMenamin J. Vaccine effectiveness in pandemic influenza-primary care reporting (VIPER): an observational study to assess the effectiveness of the pandemic influenza A (H1N1) v vaccine. Health Technol Assess 2010;14: 313-46.

27 Valenciano M, Kissling E, Cohen J-M, Oroszi B, Barret A-S, Rizzo C, et al. Estimates of pandemic influenza vaccine effectiveness in Europe, 2009-2010: results of Influenza Monitoring Vaccine Effectiveness in Europe (I-MOVE) multicentre case-control study. PLoS Med 2011;8:e1000388.

28 Jackson LA, Gaglani MJ, Keyserling HL, Balser I, Bouveret N, Fries L, et al. Safety, efficacy, and immunogenicity of an inactivated influenza vaccine in healthy adults: a randomized, placebocontrolled trial over two influenza seasons. BMC Infect Dis 2010;10:71.

29 Groenwold RH, Hak E, Hoes AW. Pooling of confounders did not induce residual confounding in influenza vaccination studies. Ann Epidemiol 2009;19:432-6.

Accepted: 22 November 2010 